\title{
Somatodendritic Secretion in Oxytocin Neurons Is Upregulated during the Female Reproductive Cycle
}

\author{
Christiaan P. J. de Kock, ${ }^{1}$ Keimpe D. B. Wierda, ${ }^{1}$ Laurens W. J. Bosman, ${ }^{1}$ Rogier Min, ${ }^{1}$ Jan-Jurjen Koksma, ${ }^{1}$ \\ Huibert D. Mansvelder, ${ }^{1}$ Matthijs Verhage, ${ }^{2}$ and Arjen B. Brussaard ${ }^{1}$ \\ Departments of ${ }^{1}$ Experimental Neurophysiology and ${ }^{2}$ Functional Genomics, Center for Neurogenomics and Cognitive Research, Vrije Universiteit \\ Amsterdam, 1081 HV Amsterdam, The Netherlands
}

During the female reproductive cycle, hypothalamic oxytocin (OT) neurons undergo sharp changes in excitability. In lactating mammals, bursts of electrical activity of OT neurons result in the release of large amounts of OT in the bloodstream, which causes milk ejection. One hypothesis is that $\mathrm{OT}$ neurons regulate their own firing activity and that of nearby OT neurons by somatodendritic release of OT. In this study, we show that OT neuron activity strongly reduces inhibitory synaptic transmission to these neurons. This effect is blocked by antagonists of both adenosine and OT receptors and is mimicked by OT application. Inhibition of soluble $\mathrm{N}$-ethylmaleimide-sensitive factor attachment protein receptor complex formation by tetanus toxin completely blocked the stimulation-induced reduction in inhibitory input, as did the calcium chelator BAPTA. During lactation, the readily releasable pool of secretory vesicles in OT cell bodies was doubled, and calcium currents were upregulated. This resulted in an increased inhibition of GABAergic synaptic transmission by somatodendritic release during lactation compared with the adult virgin stage. These results demonstrate that somatodendritic release is augmented during lactation, which is a novel form of plasticity to change the strength of synaptic transmission.

Key words: supraoptic nucleus; reproduction; $\mathrm{GABA}_{\mathrm{A}}$; capacitance measurements; oxytocin; synaptic transmission; readily releasable pool

\section{Introduction}

Many neurons change excitability during the life cycle of an organism (Summerlee, 1981; Dudek et al., 1993; Steriade et al., 1994; Leng et al., 1999; Morin, 1999; Herrmann and Knight, 2001). In general, groups of neurons shift electrical activity from being quiescent to synchronous firing or vice versa. Oxytocin (OT) neurons in the supraoptic nucleus (SON) of the hypothalamus fire at a low level during pregnancy but abruptly switch to synchronous high-frequency bursting behavior at the time of birth and during lactation in response to suckling (Summerlee, 1981; Leng et al., 1999). After the cessation of lactation, the activity of OT neurons returns to a low baseline level. Thus, the hypothalamic OT neurons display marked changes in neuronal activity with each reproductive cycle.

Intricate mechanisms underlie these changes in OT neuron excitability. Bursting of OT neurons during lactation is initiated by somatodendritic OT release within the SON (Leng et al., 1999). OT acts on receptors on nearby OT neurons, which activate calcium release from internal stores (Lambert et al., 1994). Ludwig et al. (2002) recently showed that this calcium release can stimulate and enhance OT release from dendrites. In this way, a positive feedback loop is created in which OT stimulates its own release within the SON (Neumann et al., 1993, 1994; Lambert et

\footnotetext{
Received Sept. 26, 2002; revised Jan. 15, 2003; accepted Jan. 21, 2003.

This work was supported by Netherlands Organization for Scientific Research-Earth and Life Science Grant 809.38.009. We thank Hans Lodder for technical assistance and Tinelies Busé-Pot for assistance with preparation of the dissociated cell cultures. We thank Dr. Nail Burnashev for comments on a previous version of this manuscript.

Correspondence should be addressed to Dr. Arjen B. Brussaard, Department of Experimental Neurophysiology, Center for Neurogenomics and Cognitive Research, Vrije Universiteit Amsterdam, de Boelelaan 1085, 1081 HV Amsterdam, The Netherlands. E-mail: brssrd@cncr.vu.nl.

Copyright $\odot 2003$ Society for Neuroscience $\quad 0270-6474 / 03 / 232726-09 \$ 15.00 / 0$
}

al., 1994; Leng et al., 1999). However, to initiate electrical firing activity in OT neurons to release OT into the bloodstream, additional synaptic mechanisms are required (Kombian et al., 1997). During pregnancy, strong GABAergic inhibition prohibits the OT neurons from massive activation (Brussaard et al., 1997). Just before parturition, properties of GABA synapses change because of redistribution of $\mathrm{GABA}_{\mathrm{A}}$ receptor subunits, creating receptors that lack neurosteroid sensitivity and therefore deactivate more rapidly (Brussaard et al., 1997, 1999). As a consequence, OT neurons start to escape GABA inhibition and become spontaneously active (Brussaard et al., 1997). Somatodendritic release of OT within the SON further reduces GABAergic transmission to OT neurons (Brussaard et al., 1996, 2000; Brussaard and Herbison, 2000). These changes in GABAergic synapses around parturition and during lactation cooperate to disinhibit the OT neurons and increase excitability (Brussaard et al., 1997).

Somatodendritic secretion of OT within the $\mathrm{SON}$ is presumed to be mediated by calcium-dependent exocytosis of large densecore vesicles (LDCVs) (Pow and Morris, 1989; Ludwig, 1998). In this study, we tested whether somatodendritic release shares properties with synaptic release of neurotransmitters. Microdialysis studies have shown that, during lactation, the concentration of extracellular OT within the SON increased by more than twofold (Neumann et al., 1993). Here we investigated how the process of somatodendritic release is regulated during the female reproductive cycle using membrane capacitance measurements to directly monitor somatodendritic vesicular secretion at a high temporal resolution. We found that changes in the excitability of SON neurons during the female reproductive cycle are partially based on plasticity in secretory mechanisms of retrograde messengers. 


\section{Materials and Methods}

Slice recordings. Wistar rats (male, 3-4 weeks of age; virgin female, 6-8 weeks of age; or lactating female, postparturition days 7-9; Harlan CPB, Zeist, The Netherlands) were used. Slice preparation, recording conditions, and selection criteria have been described previously (Brussaard et al., 1999). Whole-cell patch-clamp recordings were made in regions of the SON in which the abundance of oxytocinergic neurons is high (Hou-Yu et al., 1986). The recording chamber was continuously perfused with artificial CSF (ACSF) consisting of (in mM): $125 \mathrm{NaCl}, 3 \mathrm{KCl}$, $1.2 \mathrm{NaH}_{2} \mathrm{PO}_{4}, 2.4 \mathrm{CaCl}_{2}, 1.3 \mathrm{MgSO}_{4}, 25 \mathrm{NaHCO}_{3}$, and 10 glucose, carboxygenated in $5 \% \mathrm{CO}_{2}-95 \% \mathrm{O}_{2}, \mathrm{pH}$ 7.4. Voltage-clamp whole-cell recordings were made using 2-4 M $\Omega$ patch electrodes filled with intracellular medium consisting of (in mM): $141 \mathrm{CsCl}, 10 \mathrm{HEPES}, 2 \mathrm{Mg}$-ATP, and 0.1 GTP (acid free), pH 7.2, with $\mathrm{CsOH}$. No calcium chelator was used, unless mentioned otherwise. GABAergic synaptic currents were pharmacologically isolated using 6,7-dinitroquinoxaline-2,3-dione (DNQX; $20 \mu \mathrm{m}$; Sigma, St. Louis, MO) to block glutamatergic synaptic transmission. Oxytocin (1 $\mu \mathrm{M}$; Bachem, Bubendorf, Switzerland), the oxytocin antagonist [des-glycinamide ${ }^{9}, \mathrm{~d}(\mathrm{CH} 2)_{5}, \mathrm{O}-\mathrm{Me}^{-\mathrm{Tyr}^{2}}, \mathrm{Thr}^{4}, \mathrm{Orn}^{8}$ ]vasotocin $\left[\mathrm{d}\left(\mathrm{CH}_{2}\right)_{5}\right.$-OVT; $1 \mu \mathrm{M}$; Bachem], and the specific adenosine $\mathrm{A}_{1}$ receptor antagonist 8-cyclopentel-1,3-dimethylxanthine (CPT; $10 \mu \mathrm{M}$; RBI) were applied extracellularly. BAPTA (200 $\mu \mathrm{M}$; Molecular Probes, Eugene, $\mathrm{OR}$ ) and tetanus toxin (TeTx; $60 \mathrm{~nm}$ of light chain, recombinant protein) were included in the internal solution. Current-clamp recordings were made in ACSF (with no DNQX added). Internal solution consisted of (in mM): 131 K-gluconate, $9 \mathrm{KCl}, 4 \mathrm{Mg}$-ATP, $0.3 \mathrm{GTP}$, and $10 \mathrm{HEPES}$, pH 7.2, with $\mathrm{KOH}$. All experiments were performed at $33^{\circ} \mathrm{C}$.

The spontaneous IPSC (sIPSC) data obtained were analyzed off-line, after $1 \mathrm{kHz}$ filtering, with the Strathclyde computer disk recorder software of John Dempster (University of Strathclyde, Glasgow, UK). Effect on sIPSC interval was calculated during the $10 \mathrm{sec}$ interval after stimulation. Data are presented as mean \pm SEM.

Acute isolation of SON neuronal somata. Wistar rats (virgin female, 6-8 weeks of age; lactating female, postparturition days 7-9; or male, 3-4 weeks of age; Harlan CPB) were used. Slice preparation (Brussaard et al., 1999) and the cell dissociation procedure (Lambert et al., 1994) have been described previously. In general, isolated somata contained two to three initial segments of neurites. Recordings were made up to $5 \mathrm{hr}$ after isolation.

Isolated cell recordings. The recording chamber was continuously perfused with ACSF consisting of (in mM): $125 \mathrm{NaCl}, 3 \mathrm{KCl}, 1.2 \mathrm{NaH}_{2} \mathrm{PO}_{4}$, $2.4 \mathrm{CaCl}_{2}, 1.3 \mathrm{MgSO}_{4}, 25 \mathrm{NaHCO}_{3}$, and 10 glucose, carboxygenated in $5 \% \mathrm{CO}_{2}-95 \% \mathrm{O}_{2}, \mathrm{pH} 7.4$, supplemented with the potassium channel blocker 4-aminopyridine ( $1 \mathrm{~mm}$; Sigma), and, in some experiments (see Figs. $4,5,7)$, the sodium channel blocker tetrodotoxin ( $1 \mu \mathrm{M}$; Alomone Labs, Jerusalem, Israel). Whole-cell recordings were made using firepolished, Sylgard-covered, 4-5 M $\Omega$ patch electrodes. Electrodes were filled with a solution containing (in $\mathrm{mm}$ ): $102 \mathrm{CsOH}, 11 \mathrm{CsCl}, 1 \mathrm{MgCl}_{2}$, 40 HEPES, 4 Mg-ATP, 0.1 Tris-GTP, and 0.1 EGTA, pH 7.3, with $\mathrm{CH}_{3} \mathrm{COOH}$ (acetic acid). All experiments were performed at $33^{\circ} \mathrm{C}$.

The whole-cell membrane current was monitored and digitized with an EPC9 amplifier (Heka, Lambrecht, Germany). Capacitance measurements were made using Pulse software. The membrane capacitance, access conductance, and membrane conductance were calculated according to the Lindau-Neher technique, implemented as the "sine plus DC" feature of the Pulse lock-in module. A sine wave of $1 \mathrm{kHz}, 40 \mathrm{mV}$ peakto-peak, was added to a holding potential of $-70 \mathrm{mV}$. The reversal potential of the lock-in module was set to $0 \mathrm{mV}$. Before, during, and after the step depolarization, the membrane current was low-pass filtered at 3 $\mathrm{kHz}$ by the Bessel filter of the EPC9 and sampled at $10 \mathrm{kHz}$. The membrane capacitance, access conductance, and membrane conductance were calculated at $1 \mathrm{kHz}$.

Calcium currents were evoked by step depolarizations to $0 \mathrm{mV}$ starting from a holding potential of $-70 \mathrm{mV}$. Capacitance changes were calculated as the difference between the average membrane capacitance during the $100 \mathrm{msec}$ before depolarization and the membrane capacitance during the first $10 \mathrm{msec}$ of the sine wave segment after depolarization. Endocytosis was calculated between the first $10 \mathrm{msec}$ and final $10 \mathrm{msec}$ of the $100 \mathrm{msec}$ sine wave segment after depolarization. The number of calcium ions that entered the cell during a pulse was determined (after subtraction of the leak current) as the integral of the calcium current (during the depolarization as well as during the tail current): $\left(\int\left[I_{\mathrm{Ca}}(t) d t\right] /\right.$ $2 \times F) \times N_{\mathrm{A}}$, where $F$ is Faraday's constant $\left(96,485{\left.\text { coulomb } \text { mol }^{-1}\right) \text { and }}^{-1}\right.$ $N_{\mathrm{A}}$ is Avogadro's constant $\left(6.022 \times 10^{23} \mathrm{~mol}^{-1}\right)$. Leak current was determined at a holding potential of $-70 \mathrm{mV}$ during a $5 \mathrm{msec}$ interval in between the first sine wave segment and the depolarization.

In addition to capacitance changes, we also observed changes in membrane conductance (see Fig. 5A, B, bottom panels). However, these changes had very different kinetics and did not induce measurable artifacts in the capacitance trace, which is in line with the observations by Lindau and Neher (1988). For instance, in Figure 5B, the conductance remained constant after the depolarization, whereas the capacitance trace declined rapidly. On occasion, we observed a decline in conductance, whereas the corresponding capacitance trace remained constant (data not shown). Furthermore, intracellular loading with tetanus toxin to block capacitance changes during calcium influx did not change membrane conductance changes, whereas capacitance changes were blocked (see Fig. 6). Therefore, we conclude that cross-talk between capacitance and conductance traces was minimal.

\section{Results}

\section{Postsynaptic secretion of OT and adenosine inhibits presynaptic GABA release}

To test whether OT neurons secrete OT in the SON, and thereby reduce their own synaptic inhibition, we first stimulated OT neurons electrically with short trains of depolarizations $[0 \mathrm{mV}$ for $100 \mathrm{msec}, 20$ times at $2 \mathrm{~Hz}$, in conformance with the protocol used by Kombian et al. (1997)] and monitored sIPSC amplitude and frequency. For ethical reasons, we used juvenile male rats [postnatal day 21 (P21) to P28] in the first part of the study (Figs. 1-3) (see Discussion).

Electrical stimulation of the postsynaptic OT neuron decreased the amplitudes of sIPSCs and decreased the sIPSC frequency in $\sim 50 \%$ of the recordings. In cells that showed a significant increase, sIPSC intervals increased by $61.3 \pm 8.4 \%(n=56)$ (Fig. 1). In some recordings, sIPSC frequency did not completely recover within the first $60 \mathrm{sec}$. However, in all cells, we observed complete recovery within $180 \mathrm{sec}$. The effect on sIPSC frequency suggests that a presynaptic locus is involved in the effect. Either the firing frequency of GABA neurons was reduced or the GABA release properties were changed (or both). In either case, the postsynaptic depolarizations affect the presynaptic GABAergic neuron, which suggests the action of a retrograde messenger.

We repeated the above experiment in the presence of the OT antagonist $\mathrm{d}\left(\mathrm{CH}_{2}\right)_{5}$-OVT $(1 \mu \mathrm{M})$, which is a modified form of vasotocin that specifically blocks OT receptors. In two of eight cells, application of vasotocin reduced the average interval between sIPSCs before electrical stimulation by itself (data not shown), indicating that OT may have been present extracellularly in these slices. After a steady-state sIPSC frequency was reached, the effect of postsynaptic depolarization on the sIPSC interval was reduced (Fig. 2A,B), indicating that OT is mediating at least part of the retrograde signaling.

Exogenous OT $(1 \mu \mathrm{M})$ application increased the GABAergic sIPSC interval in six of eight cells (Fig. 2C). The two insensitive cells most likely were vasopressinergic neurons. Together, these results show that postsynaptically released OT mediates the effect on sIPSC interval. However, OT may not be the only retrograde messenger given the partial block by vasotocin.

To test whether OT is the only retrograde messenger, we first saturated the OT effect. Applying the saturating concentration of $5 \mu \mathrm{M}$ OT (Brussaard et al., 1996), sIPSC intervals were increased by $54.7 \pm 16.4 \%$ (Fig. $2 \mathrm{D}$ ). Electrical stimulation in the presence 

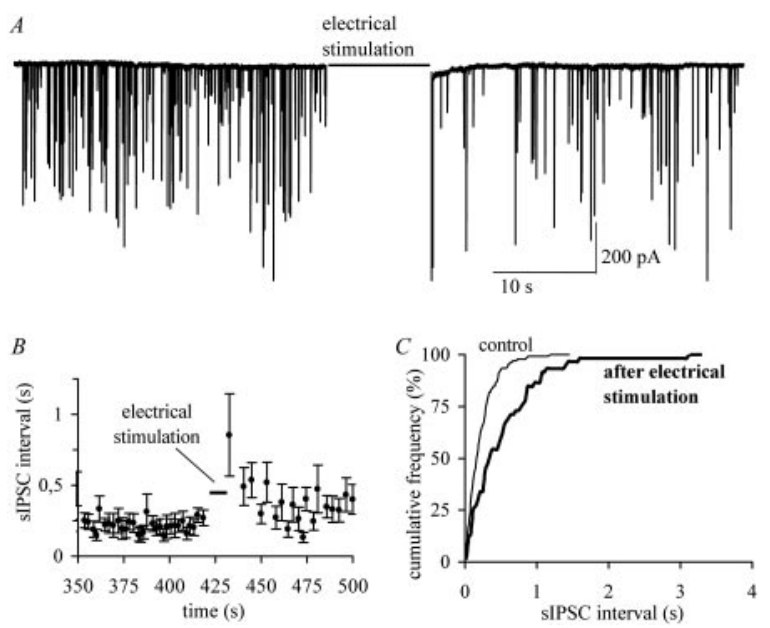

Figure 1. Postsynaptic stimulation inhibits presynaptic GABA release. A, Electrical stimulation of postsynaptic SON neurons from slices of juvenile rats (P21-P28) induced an increase in the interval between spontaneous GABAergic events indicative of the inhibition of presynaptic GABA release (example trace, $n=56$ ). A $2 \mathrm{~Hz}$ stimulation protocol was used (see Materials and Methods). B, Electrical stimulation induced instantaneous but short-term depression of sIPSC frequency. C, Corresponding cumulative frequency plot showing that, after electrical stimulation, short intervals occurred less frequently.
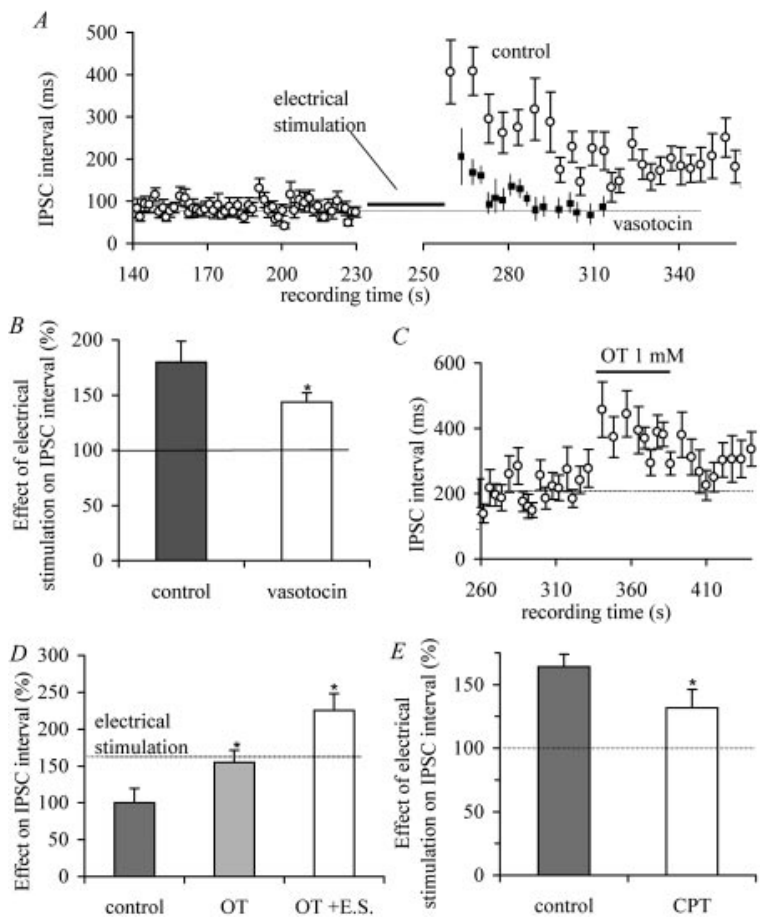

Figure 2. Corelease of $0 T$ and adenosine inhibits presynaptic GABA release. $A$, In the presence of the $0 T$ receptor antagonist vasotocin $(1 \mu \mathrm{m})$, the effect of electrical stimulation on GABAergic sIPSC frequency was partly, although significantly, blocked (paired ttest; $p<0.05 ; n=8$ ). $\bigcirc$, Control; $\mathbf{\square}$, vasotocin. $B$, Summary of the effect of postsynaptic stimulation after block of the $0 T$ receptor. Note that after blockade, the effect of postsynaptic OT release was reduced but still present, indicating potential release of an additional retrograde messenger. C, Bath application of $0 \mathrm{~T}(1 \mu \mathrm{M})$ mimicked the effect of postsynaptic depolarization on sIPSC frequency, inducing an increase in sIPSC interval (paired $t$ test; $p<0.01 ; n=6$ ). D, Application of saturating concentrations of $0 \mathrm{~T}(5 \mu \mathrm{m})$ induced an increase in sIPSC interval. The dotted line indicates the level of electrical stimulation in the absence of OT application. Electrical stimulation (E.S.) in the presence of 0 T induced an additional increase in sIPSC interval (paired ttest; $p<0.01 ; n=3$ ). E, Under control conditions, electrical stimulation resulted in increase in the interval of GABAergic sIPSCs. In the presence of the specific adenosine antagonist CPT $(10 \mu \mathrm{m})$, the effect of electrical stimulation was significantly reduced (Mann-Whitney; $p<0.05 ; n=$ 12). Asterisks indicate significant difference from control, and in D, OT + E.S. from OT.
A
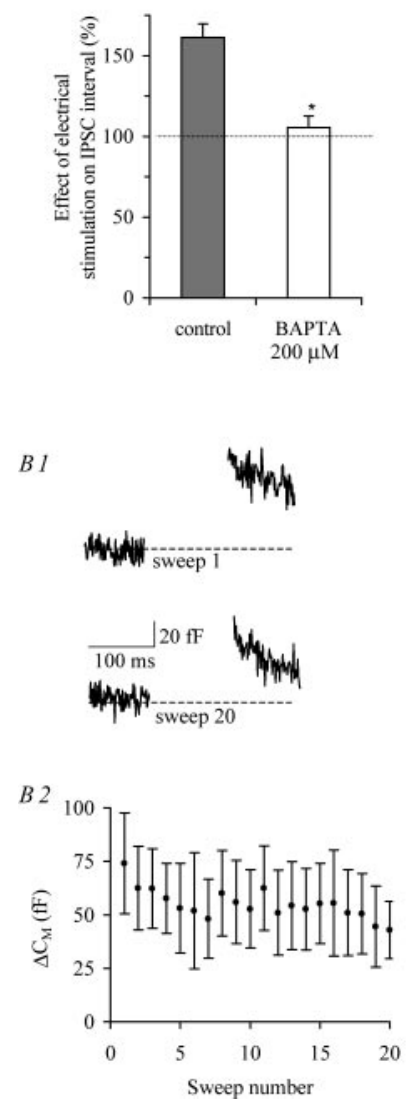
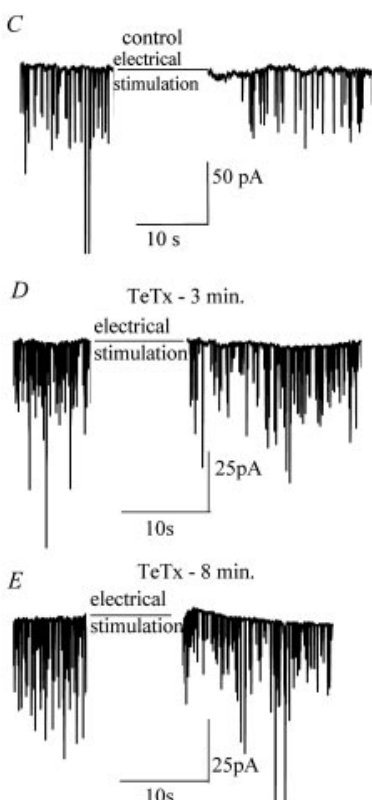

F

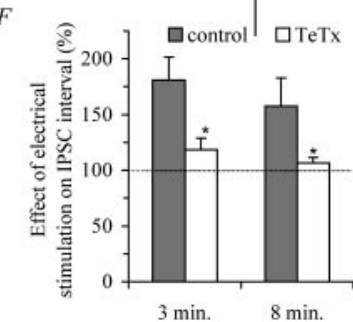

Figure 3. SNARE complex mediates somatodendritic secretion of OT and adenosine. $A$, The depression of sIPSC frequency was blocked on loading the postsynaptic SON neuron with 200 $\mu$ M BAPTA (unpaired $t$ test; $p<0.01 ; n=10$ ). B1, Capacitance measurements on isolated neurons from juvenile rats (P21-P28) to directly record somatodendritic LDCV release during the same $2 \mathrm{~Hz}$ depolarization protocol applied in slices (averaged capacitance changes during first and 20th sweep; $n=4)$. The dashed lines indicate cell capacitance before stimulation. B2, Depolarization induced capacitance changes in isolated neurons from juvenile rats. C, Postsynaptic depolarization decreased the frequency of GABAergic sIPSC in brain slices. D, E, Perfusion of the postsynaptic neuron for $3(D)$ and $8(E)$ min with the (membrane-impermeable) active subunit of the clodistrial protein TeTx ( $60 \mathrm{~nm})$ to cleave synaptobrevin decreased the effect of postsynaptic stimulation. F, Summary of the effect of TeTx on the interval of GABAergic sIPSCs. In the presence of TeTx ( $3 \mathrm{~min})$, the effect of electrical stimulation on sIPSC interval was reduced (ANOVA; $p<0.05 ; n=12$ ). Prolonged exposure to TeTx ( $8 \mathrm{~min}$ ) completely abolished the effect of electrical stimulation $(n=3)$. Dotted lines represent IPSC interval before electrical stimulation. Asterisks indicate significant difference from control.

of $5 \mu \mathrm{M}$ OT further increased the sIPSC interval to $125.5 \pm 22.5 \%$ (Fig. 2D), confirming that a second retrograde messenger is involved. A candidate for a second retrograde messenger is adenosine (Oliet and Poulain, 1999). We depolarized the postsynaptic OT neuron in the presence of the adenosine $\mathrm{A}_{1}$ receptor antagonist CPT $(10 \mu \mathrm{M})$. This reduced the effect of electrical stimulation significantly (from $64.1 \pm 10.1$ to $31.7 \pm 14.6 \%$ ) (Fig. $2 E$ ).

Somatodendritic release is calcium and soluble $\mathrm{N}$-ethylmaleimide-sensitive factor attachment protein receptor dependent

Intracellular application of the calcium chelator BAPTA (200 $\mu \mathrm{M})$ completely prevented the reduction of sIPSC frequency induced by electrical stimulation (Fig. $3 A$ ). The increase in sIPSC interval was reduced from $61.3 \pm 8.4(n=56)$ to $5.5 \pm 7.0 \%(n=$ $10)$ in the presence of BAPTA. Hence, somatodendritic release of 
OT and adenosine is calcium dependent, as is synaptic secretion of neurotransmitters.

To monitor somatodendritic secretion by OT neurons more directly, we performed capacitance measurements on acutely dissociated SON somata. When vesicles fuse with the cell membrane to release their contents in the extracellular space, the capacitance of the whole-cell membrane increases (usually $<0.1 \%$ ). These tiny increases can be captured with high sensitivity and temporal resolution with a variant of the voltage-clamp method called capacitance measurements (Neher and Marty, 1982). During the same $2 \mathrm{~Hz}$ depolarization protocol used in the slice recordings, changes in the surface membrane area were induced (Fig. 3B2) $(n=4)$, leading to an average capacitance change $\left(\Delta C_{\mathrm{M}}\right)$ of $74.0 \pm 23.5 \mathrm{fF}$ during the first depolarization-induced calcium influx (Fig. 3B1, top panel) and gradually decreasing to $42.9 \pm$ $13.4 \mathrm{fF}$ during the 20th sweep (Fig. 3B1, bottom panel). The decrease in vesicle release on repeated step depolarizations may be explained by both calcium channel inactivation and depletion of the readily releasable pool (RRP).

Synaptic release of neurotransmitters and neuropeptides from secretory vesicles depends on the soluble $\mathrm{N}$-ethylmaleimidesensitive factor attachment protein receptor (SNARE) complex. To test whether the SNARE complex is also involved in the secretion of retrograde messengers by OT neurons, we applied TeTx, which specifically cleaves synaptobrevin, a component of the SNARE complex (Link et al., 1992; Schiavo et al., 1992). Under control conditions, postsynaptic electrical stimulation after 3 min of recording increased the interval of GABAergic sIPSCs by $80.3 \pm 20.8 \%$ (Fig. $3 C)(n=$ 12). Loading the cell with $\mathrm{TeTx}(60 \mathrm{nM})$ for 3 min attenuated the effect of postsynaptic electrical stimulation to $18.3 \pm 10.5 \%$ (Fig. $3 D$ ). Prolonged application of TeTx $(8 \mathrm{~min})$ completely abolished the effect of electrical stimulation on the sIPSC interval (Fig. 3E). Under control conditions, the sIPSC interval was still increased by $57.7 \pm 21.7 \%$ after electrical stimulation at $8 \min (n=3)$. Thus, somatodendritic release by OT neurons depends on the SNARE complex.

\section{Somatodendritic LDCV release is regulated during lactation}

To study somatodendritic secretion during the female reproductive cycle, we used capacitance measurements on isolated somata from adult female rats. In neurons from virgin females, a depolarization in the order of the duration of an action potential (AP; $2 \mathrm{msec}$ ) induced a $\Delta C_{\mathrm{M}}$ of $16.6 \pm 3.5 \mathrm{fF}$ (Fig. $\left.4 A\right)(n=13)$. Given the capacitance of a single LDCV in SON terminals in the posterior pituitary [412 $\pm 16 \mathrm{aF}$ (Klyachko and Jackson, 2002)], this suggests that $\sim 40 \pm 8 \mathrm{LDCVs}$ can be released from the somatodendritic compartment by a single 2 msec depolarization. Increasing the length of the stimulus duration resulted in increased calcium influx and consequently in enhanced exocytosis (Fig. $4 A)(n=13)$. Capacitance changes were not linearly related to stimulus duration, most likely because of calcium current inactivation and the depletion of the RRP (Fig. $4 D$, but see below). These results show that vesicle secretion can be induced from the isolated somatodendritic compartment of SON neurons. This somatodendritic secretion is also calcium dependent, because no capacitance changes are induced in calcium-free $\operatorname{ACSF}(n=3$; data not shown).

In the SON, many cellular processes are under control of the female reproductive cycle (Theodosis et al., 1994; Hatton, 1997; Brussaard and Herbison, 2000). To examine whether somatodendritic release is regulated during lactation, acutely isolated SON somata of lactating females on days 7-9 (lact. 7-9) were compared with SON somata of virgin females. Somata from lact.
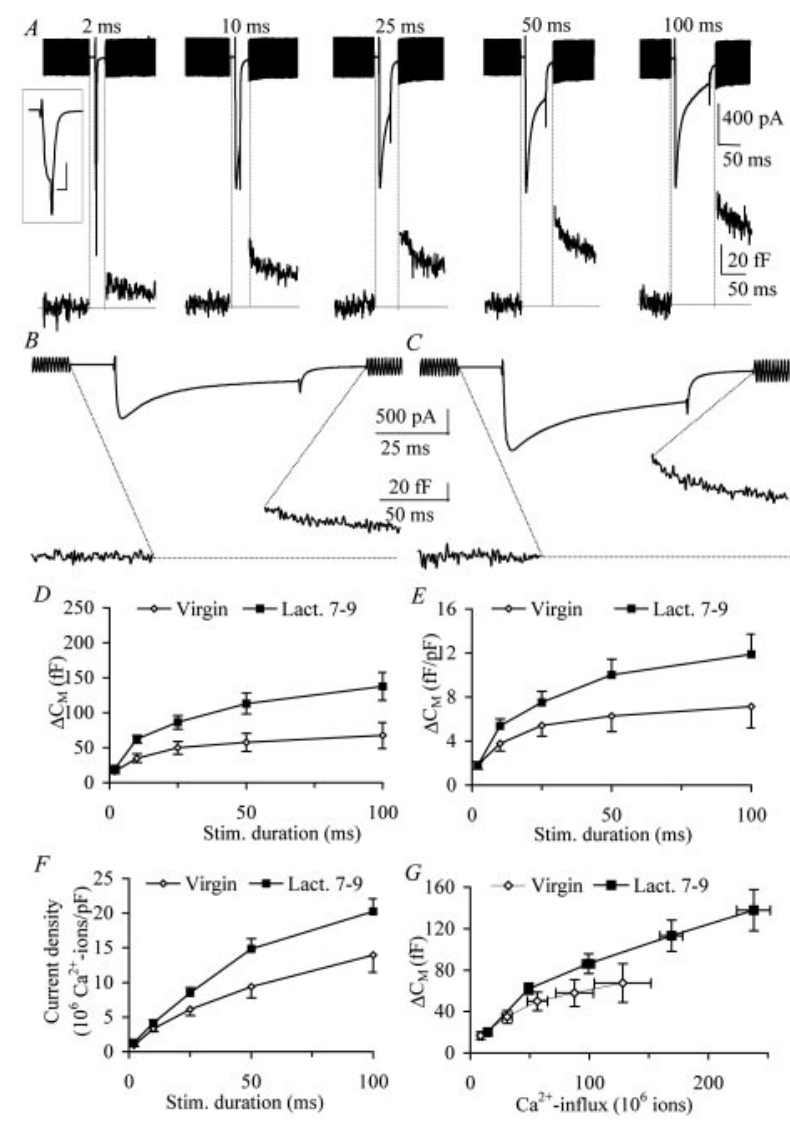

Figure 4. Somatodendritic LDCV release is upregulated during lactation. A, Depolarizationinduced calcium current and corresponding membrane capacitance changes in acutely dissociated magnocellular neurons from supraoptic nucleus from adult female virgin animals (6-8 weeks of age; averaged trace; $n=13$ ). Calibration, inset, $400 \mathrm{pA}, 2 \mathrm{msec}$. B, Calcium current and corresponding membrane capacitance during a $50 \mathrm{msec}$ depolarization from isolated neurons in adult female virgin animals ( $6-8$ weeks of age; averaged trace). C, Analogous responses in neurons from lactating animals (lact. 7-9; averaged trace). Note both the increased amplitude of the calcium current and increased exocytotic activity in neurons from lactating animals. D, Membrane capacitance responses were significantly increased for all depolarization durations beginning from $10 \mathrm{msec}$ in isolated neurons from lactating animals (virgin, $\diamond, n=13$; lact. $7-9, \mathbf{\square}, n=17$; two-way ANOVA; $p<0.01$ ). E, Capacitance changes were corrected for cell size. After correction, a significantly increased exocytotic activity was still observed (two-way ANOVA; $p<0.01) . F$, The integral of the calcium current was calculated to produce the absolute $\mathrm{Ca}^{2+}$ influx (see Materials and Methods). The calcium influx was corrected for cell capacitance to determine the current density. Note that the current density in neurons from lactating animals is significantly upregulated compared with neurons from virgin animals (two-way ANOVA; $p<0.01$ ), indicative of an increased functional expression of voltage-dependent calcium channels. $G$, Capacitance changes in response to long stimulus durations are limited by the size of the RRP and not calcium channel inactivation. Note that the maximum level in neurons from lactating animals is enhanced, indicating a larger RRP.

7-9 animals showed significantly increased secretory responses to all depolarization durations of $\geq 10 \mathrm{msec}$ (Fig. $4 B-D$ ). In addition to increased exocytosis during lactation, the total cell membrane capacitance in lactating animals was increased by $40 \%$ $(n=17$; data not shown). To test whether the observed increased exocytotic response during lactation results from an increase in total cell surface, we normalized capacitance changes to the cell capacitance. The capacitance increase per unit surface area in lactating animals was still significantly larger compared with neurons from virgin animals (Fig. $4 E$ ), indicating that exocytosis is upregulated in addition to the larger cell size.

The increase in exocytosis during the reproductive cycle was accompanied by an increase in amplitude of the calcium influx 
(Fig. 4B,C). At 50 msec stimulus duration, the peak amplitude increased from $-1133 \pm 165 \mathrm{pA}(n=13$, virgin $)$ to $-1791 \pm 101$ pA $(n=17$, lact. 7-9). The slow-inactivating calcium current (calcium current measured $2 \mathrm{msec}$ before repolarization) increased from $-334 \pm 72 \mathrm{pA}(n=13$, virgin $)$ to $-707 \pm 43 \mathrm{pA}$ ( $n=17$, lact. 7-9; data not shown). As a result, the total influx of calcium ions is larger in lactating animals [i.e., more calcium ions flow into the cell per surface area (Fig. $4 F$ )]. These data indicate that, during lactation, the density of voltage-gated calcium channels is increased or the properties of the calcium channels have changed to allow more calcium entry.

During long stimulus durations, exocytosis saturates (Fig. $4 D$ ). This could be attributable to either depletion of the RRP of LDCVs or calcium channel inactivation. Plotting the capacitance changes against the amount of calcium influx showed that increasing calcium influx with longer depolarizations does not lead to proportional increases in exocytosis (Fig. 4G). This relationship also reaches a maximum. Thus, most likely, at long stimulus durations, the RRP depletes while calcium influx still continues.

We used the level of capacitance changes at the $100 \mathrm{msec}$ stimulus duration to estimate the RRP in SON neurons. The average $\Delta C_{\mathrm{M}}$ in neurons from virgin animals during a $100 \mathrm{msec}$ depolarization $(67.5 \pm 16.5 \mathrm{fF})$ implies an RRP size of $164 \pm 40$ LDCVs. In lactating animals, $\Delta C_{\mathrm{M}}$ induced by $100 \mathrm{msec}$ depolarizations (137.68 $\pm 19.9 \mathrm{fF}$ ) implies a much larger RRP size (334 \pm 48 LDCVs), indicating a $104 \%$ upregulation of the amount of releasable vesicles. Thus, an increased pool of LDCVs that can be released rapidly together with more calcium entry will lead to an increased amount of secretion per action potential, and will strongly contribute to increased levels of OT in the SON after parturition.

\section{Endocytosis is proportionally regulated during the reproductive cycle}

After the initial increase in $C_{\mathrm{M}}$, in approximately one-half of the neurons from virgin animals, the capacitance traces declined (Fig. 5A,B). Most likely, this reflects rapid endocytosis (Mansvelder and Kits, 1998). In both virgin and lactating animals, rapid endocytosis had a time constant of $\sim 30 \mathrm{msec}$ (Fig. $5 C$ ). Slow endocytosis also occurred, and typically, $\Delta C_{\mathrm{M}}$ returned to baseline within $5 \mathrm{sec}$ (data not shown). The amount of retrieved membrane by rapid endocytosis in virgin neurons was linearly dependent on the amount of exocytosis, suggesting that the history of secretory activity determines the amplitude of endocytosis (Fig. 5E, bracket line). In lactating animals, we almost always observed rapid endocytosis ( 15 of 17 cells). In these animals, the amplitude of fast endocytosis was increased (Fig. 5D) and was also proportional to the exocytotic activity (Fig. $5 E$, solid line). We conclude that the increase in exocytotic activity observed during lactation was compensated for by an increase in rapid endocytosis, possibly to prevent large changes in cell surface areas.

\section{Synaptobrevin mediates LDCV release in SON neurons}

TeTx blocks the effect of electrical stimulation on inhibitory synaptic transmission in the SON slice (Fig. $3 C-F$ ). In isolated neurons, loading the cell through the patch pipette with $200 \mathrm{~nm} \mathrm{TeTx}$ substantially blocked depolarization-induced capacitance changes depending on application length (Fig. 6C,D), whereas inward currents were not affected (Fig. $6 A-D$, insets). Prolonged exposure to TeTx (5-10 min) completely blocked LDCV release $(n=4$; data not shown). Together, these results show that soma-
A
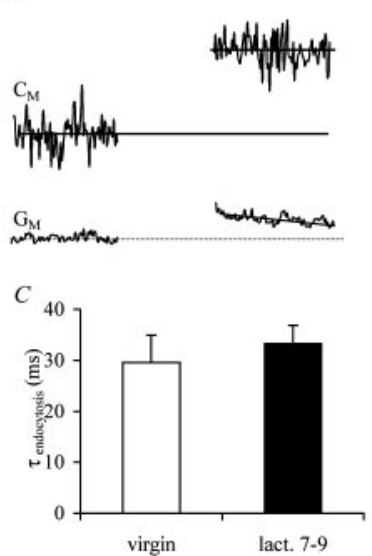

B
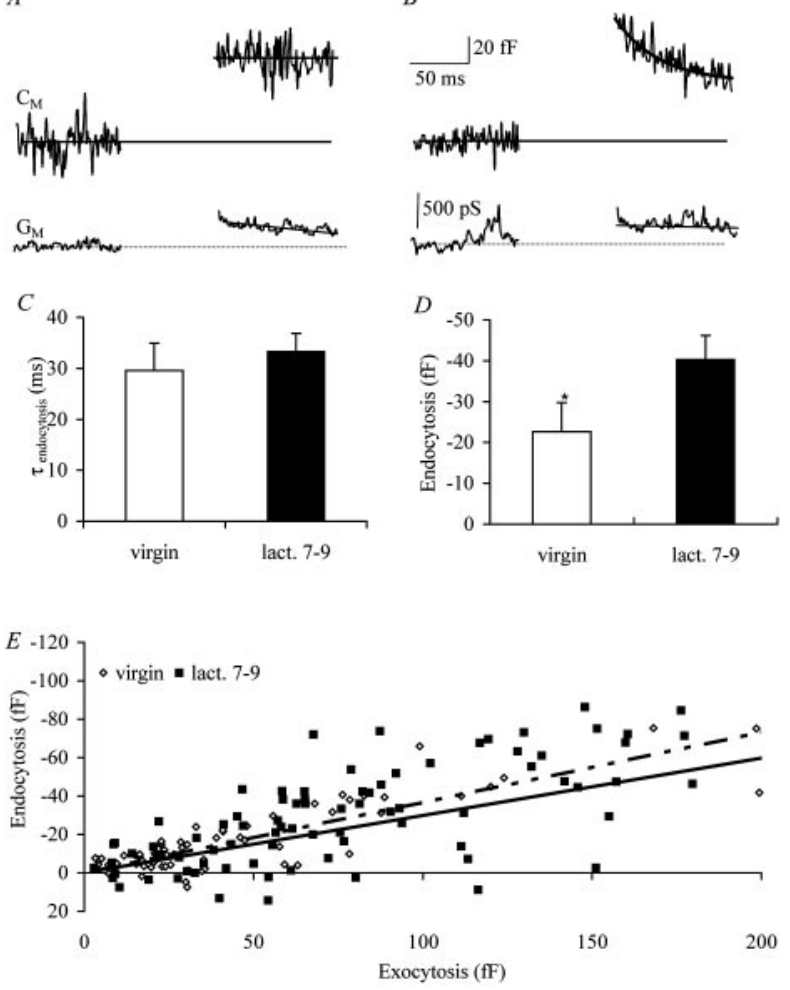

Figure 5. Endocytosis is linearly dependent on exocytosis. $A$, Example trace of a neuron from a virgin animal after 50 msec of depolarization with an apparent lack of fast endocytosis. $B$, Example trace with clear presence of fast endocytosis. The solid line is an exponential fit with a time constant of $29.6 \pm 5.4 \mathrm{msec}(n=7)$. C, Time course of fast endocytosis is not regulated during female reproductive cycle (virgin, $n=7$; lact. $7-9, n=15$ ). $D$, The amplitude of endocytosis is increased during lactation stage, compensating for increased levels of secretion (endocytosis calculated in all cells; virgin, $n=13$; lact. 7-9, $n=17 ; p<0.05$ ). Asterisk indicates significant difference from lact. 7-9. E, Endocytosis is linearly dependent on exocytosis for neurons of both virgin and lactation stage (virgin, dashed-dotted line; lact. 7-9, solid line).

todendritic release of OT and adenosine is dependent on synaptobrevin and the SNARE complex.

\section{Somatodendritic release can be evoked by single action potentials}

To study whether a single action potential can evoke LDCV release, we recorded action potentials from SON neurons in slices from female lactating rats (postparturition day 7 ). The recorded action potential was used as voltage template in capacitance recordings from dissociated neurons (Fig. 7). To apply the sine wave at a holding membrane potential of $-70 \mathrm{mV}$, as is necessary for capacitance recordings, the action potential template was adjusted to start at $-70 \mathrm{mV}$. In dissociated neurons of lact. 7-9 rats, a single action potential evoked capacitance changes of $18.59 \pm$ $3.1 \mathrm{fF}$, which implies the release of $45 \pm 8$ vesicles (Fig. $7 \mathrm{~B}, \mathrm{C}$ ). This is not significantly different from the response to a square pulse of $2 \mathrm{msec}$ ( $49 \pm 8$ vesicles). Analysis of the amount of calcium influx also shows that there is no difference between the single action potential and the $2 \mathrm{msec}$ square depolarization (Fig. 7C).

In addition to single action potentials, we also recorded spontaneous bursts of action potentials. The action potential frequency within bursts was variable but was typically in the order of $20 \mathrm{~Hz}$. We used the first action potential up to a train of four action potentials as voltage template in dissociated neurons of lact. 7-9 neurons. The amount of capacitance change increased 


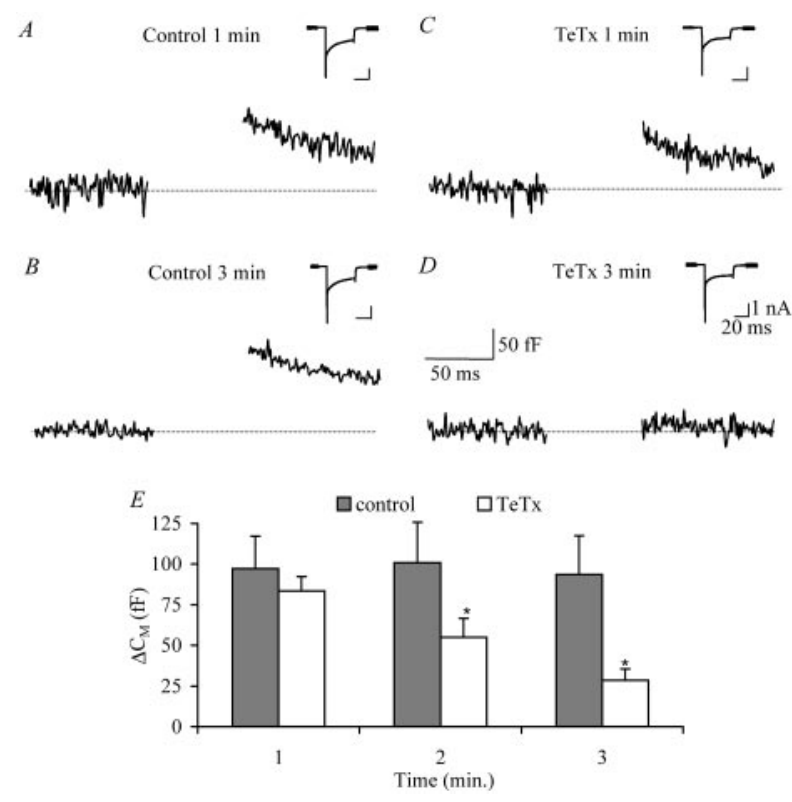

Figure 6. SNARE complex mediates somatodendritic LDCV release in SON neurons. A, Membrane capacitance change (example trace) in response to $40 \mathrm{msec}$ depolarization in neurons from lactating animals after $1 \mathrm{~min}$ in the whole-cell configuration. The inset shows the corresponding inward (sodium and calcium) currents. B, Corresponding capacitance change after 3 min of recording. Note the constant level of release during the experiment. The inset shows that inward currents were also constant during the experiment. C, Capacitance change at 1 min after establishing whole-cell configuration (example trace) in the presence of $200 \mathrm{~nm}$ of the active subunit of TeTx to cleave synaptobrevin. The inset shows that there was no effect on the inward currents. D, Capacitance change at 3 min after establishing whole-cell configuration (example trace), which shows the increased inhibition by TeTx in time. The inset shows that the effect of TeTx was specific for the capacitance changes, without effecting inward currents. E, Summary of the effect of TeTx on somatodendritic release in SON neurons. Note that under control conditions $(n=10), \mathrm{LDCV}$ release was constant, whereas in the presence of $\operatorname{TeTx}(n=9)$, release was significantly inhibited (two-way ANOVA; $p<0.01$ ). Asterisks indicate significant difference from control at each time point.

with increasing number of action potentials (Fig. 7D). These results show that vesicle secretion can be induced from the isolated somatodendritic compartment of SON neurons by single action potentials.

Upregulation of somatodendritic release increases GABAergic synaptic depression

Above, we showed that, during the reproductive cycle, somatodendritic release is increased in isolated neurons (Fig. 4). To study the consequence of this increase for retrograde signaling in the SON, we studied the effect of electrical stimulation during different stages of the reproductive cycle. In slices from virgin animals, a significant increase in sIPSC interval was observed after electrical stimulation in four of nine cells $(123.6 \% \pm 16.3$; $n=9$ ) (Fig. 8). Afterward, the sIPSC interval returns to baseline values within $40 \mathrm{sec}$. In slices from lactating females, electrical stimulation significantly increased sIPSC interval in six of eight cells, and the overall effect was much larger than in virgin animals $(158.2 \% \pm 19.4 ; n=8$; two-way ANOVA; $p<0.05)$ (Fig. $8 B, C)$. The sIPSC interval again returned to baseline values within $40 \mathrm{sec}$ after electrical stimulation. We conclude that increased inhibition of GABAergic inputs during lactation is caused by upregulation of somatodendritic release of retrograde messengers.

\section{Discussion}

In this study, we identified a new mechanism that regulates somatodendritic release during the female reproductive cycle. Elec-
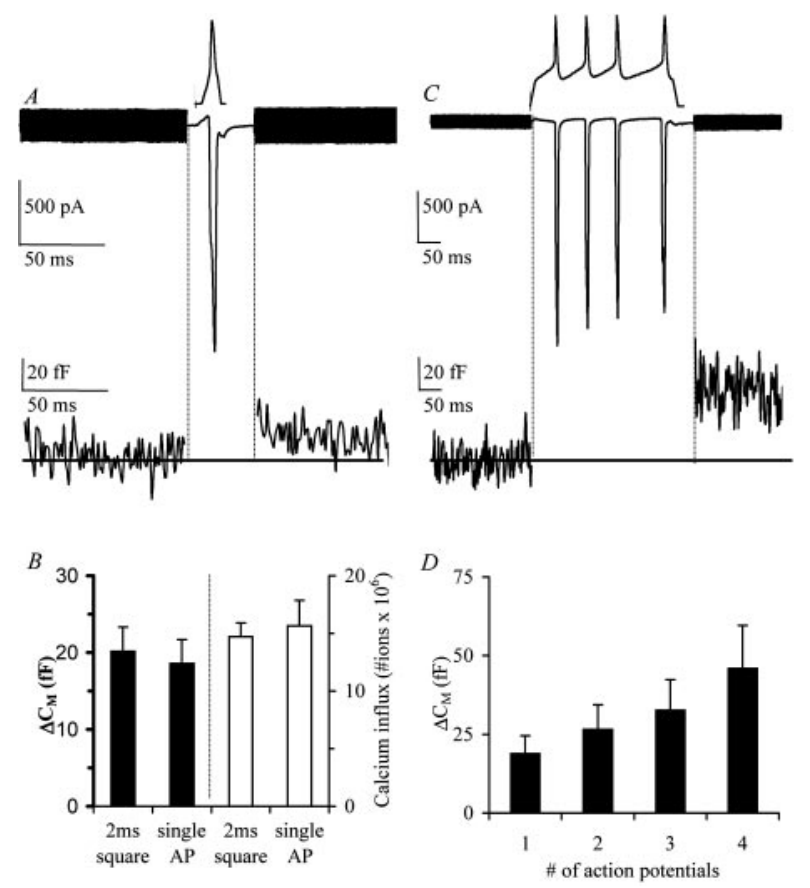

Figure 7. Somatodendritic release can be evoked by a single action potential. A, Calcium current and corresponding membrane capacitance during a single action potential from isolated neurons in adult lactating females (lact. $7-9$, averaged trace, $n=7$ ). The voltage template is shown above the current trace. $B$, Summary of the capacitance changes and calcium influx in response to square pulses of 2 msec compared with single action potentials. C, Calcium current and corresponding membrane capacitance during a train of action potentials from isolated neurons in adult lactating females (lact. 7-9, averaged trace, $n=4$ ). The voltage template is shown above the current trace. $D$, Summary of the response to single and trains of action potentials $(n=4)$.

trical activity in OT neurons in slices causes the release of OT and adenosine from the somatodendritic compartment, which inhibits GABAergic inputs to these neurons. Somatodendritic release resembles synaptic neurotransmitter release in that it is calcium dependent and mediated by the SNARE complex. However, within the SON, OT and adenosine are not released from synaptic contacts. Isolated somata from the SON show exocytosis in response to electrical activity and calcium influx, which is also mediated by the SNARE complex. During lactation, the readily releasable pool of LDCVs in SON neurons is upregulated to twice its size in virgin animals, and the calcium channel activity is also strongly upregulated to allow substantially more calcium entry. The facilitation of somatodendritic release results in increased inhibition of GABAergic synaptic transmission during lactation compared with the adult virgin stage. Thus, at a time when OT neurons need to be very active as an ensemble, twice as much of the retrograde messengers can be released rapidly to reduce the inhibitory synaptic input to the OT neurons.

In the first part of this study, we used young male rats to study the biophysical mechanisms of somatodendritic release in the SON. In males, OT from the SON acts also as a hormone and is involved in both steroid metabolism and ejaculation (Ivell et al., 1997). Although release of OT in the SON of male animals is less well studied, we hypothesize that somatodendritic release of OT in males will also recruit neighboring OT neurons to facilitate synchronous firing behavior. Moreover, modulation of amplitude of postsynaptic GABAergic events by somatodendritic OT release is comparable between juvenile males and females (Brussaard et al., 1996). Therefore, we feel justified in using males to study the biophysical properties of somatodendritic OT release. 

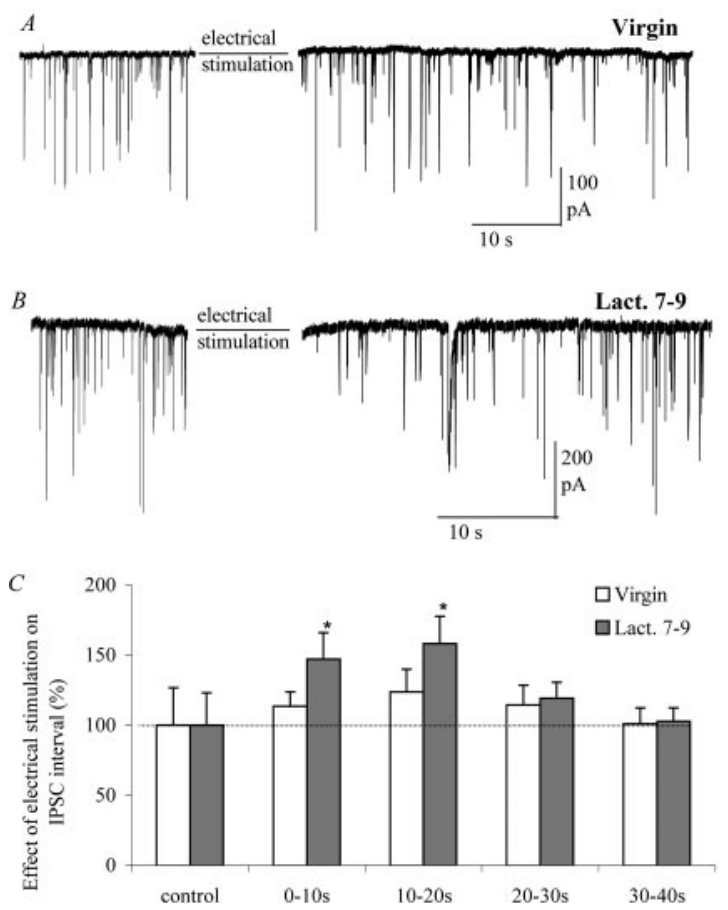

Figure 8. Increased inhibition of GABAergic inputs during lactation. A, Electrical stimulation of postsynaptic SON neurons from slices of adult virgin animals ( $6-8$ weeks of age) induced a moderate increase in sIPSC interval (example trace, $n=9$ ). B, Electrical stimulation of postsynaptic SON neurons of lactating days 7-9 animals had a much larger effect on sIPSC interval (example trace, $n=8$ ). C, Summary of the effect of electrical stimulation on sIPSC interval in neurons from virgin animals versus neurons from lactating females. The increase in interval is significantly larger at the lactating stage (two-way ANOVA; $p<0.05$ ) compared with the nonreproductive stage.

\section{Somatodendritic secretion of retrograde transmitters}

Somatodendritic release has been reported in other brain areas as well. For instance, in the substantia nigra, dopamine is released within the nucleus (Cheramy et al., 1981; Jaffe et al., 1998). Interestingly, dopamine acts on presynaptic GABAergic terminals (Radnikow and Misgeld, 1998). Also, in the raphe nucleus, somatodendritic release of serotonin (5-HT) affects presynaptic release of GABA (Bagdy et al., 1998; Bunin and Wightman, 1998; Liu et al., 2000). Although it is unknown whether in these nuclei changes in dopamine and serotonin release underlie changes in excitability and synchronous firing, retrograde signaling that leads to short-term synaptic plasticity may be a general concept.

There may exist two ways by which dopamine is released from the somatodendritic compartment within the substantia nigra. One is by the release of vesicles, which is calcium dependent (Jaffe et al., 1998). The other way is through the reversal of dopamine carriers (Falkenburger et al., 2001). In the raphe nucleus, it is not clear whether vesicular release is contributing to somatodendritic release, but the occurrence of carrier-mediated release of serotonin has been reported previously (Bagdy et al., 1998). Increases in membrane capacitance in response to calcium influx have been found in isolated somata of dorsal root ganglia (Huang and $\mathrm{Ne}$ her, 1996), indicating that, in these neurons, somatic release is vesicular and calcium dependent. However, in DRG neurons, somatic release takes place in the order of seconds. We found that, in OT neurons in the SON, somatodendritic release can occur on a millisecond time scale. Moreover, we found that inhibition of SNARE complex formation blocks somatodendritic release in slices as well as in isolated somata. Together, this strongly argues in favor of a vesicular mechanism of release of OT and adenosine, which is in line with the findings of Pow and Morris (1989) who showed with electron microscopy vesicular release from OT somata. However, oxytocin-immunoreactive terminals on oxytocin neurons may be present in the supraoptic nucleus (Theodosis, 1985). Therefore, we cannot exclude the possibility that part of the oxytocin-mediated effect in slices could be attributable to recurrent axon collaterals. Nevertheless, this possibility can be ruled out for the experiments performed on acutely isolated neurons, in which we show that a single action potential is sufficient to evoke release of $\sim 40$ LDCVs, suggesting that a single somatic and/or back propagating action potential in the dendrite is sufficient to induce the release of LDCVs.

Recently, Ludwig et al. (2002) reported that calcium release from internal stores activates and enhances OT release from dendrites. However, they also reported that neural stalk stimulation could cause OT release from the nerve terminals with little or no release from the dendrites. In contrast, we find that single action potentials are sufficient to induce membrane capacitance changes in isolated SON somata. In the study by Ludwig et al. (2002), rats were anesthetized with urethane during the experiment. This anesthetic strongly augments GABAergic transmission throughout the CNS, and because OT neurons receive GABAergic inputs on somata and dendrites, this will increase inhibition of the soma and dendrites. Therefore, stalk stimulation may result in an action potential traveling to the pituitary terminals, but the antidromic spike may not reach the soma and dendrites, and thus fail to induce release from these compartments.

Furthermore, we used capacitance measurements of single neurons with very high sensitivity and temporal resolution, whereas Ludwig et al. (2002) used microdialysis of large parts of the SON with a temporal resolution of $30 \mathrm{~min}$. It is not likely that microdialysis would detect OT released from single neurons by single spikes within milliseconds after the spike. This detection is also hindered by endogenous protease activity, which breaks down extracellular OT (Kombian et al., 1997).

\section{Regulation of somatodendritic release during lactation}

During the female reproductive cycle, the AP in OT neurons increases in duration (Armstrong et al., 2002). We found that, during lactation, calcium channel activity is upregulated, which allows more calcium entry. It is likely that this calcium channel upregulation contributes to AP broadening by introducing a pronounced calcium shoulder in the falling phase of the AP (Armstrong et al., 2002). Lengthening of the AP will result in more calcium entry and more exocytosis, because we found that increasing the depolarization duration beyond $2 \mathrm{msec}$ resulted in enhancement of $\Delta C_{\mathrm{M}}$. We also found that exocytosis is increased during lactation. This could be explained by regulation of coupling between calcium channels and vesicles. However, the full inhibition of the reduction of sIPSC frequency by $0.2 \mathrm{~mm}$ BAPTA most likely does not suggest a very tight coupling between calcium channels and vesicles. Therefore, we conclude that the number of vesicles is increased, causing the RRP in neurons from lactating females to be twice as large as in virgin animals (Fig. 4). With longer APs and sustained electrical activity (Leng et al., 1999) of OT neurons during lactation, a larger RRP will prevent fast depletion of releasable vesicles with OT and adenosine. This results in increased inhibition of GABAergic synaptic transmission during lactation compared with that of the virgin stage (Fig. 8).

Extracellular concentrations of OT and adenosine may not be affected only by the upregulation of release on lactation. During the reproductive cycle, structural plasticity in the hypothalamic 
system leads to retraction of glial cells and hypertrophy of neurons (Theodosis et al., 1986; Theodosis and Poulain, 1993; Hatton, 1997). Oliet et al. (2001) showed that regulation of synaptic release of glutamate within the $\mathrm{SON}$ is under control of metabotropic glutamate receptors and proposed that this type of retrograde signaling is sensitive to the extent of glial withdrawal. Thus, somatodendritic secretion of OT and adenosine may also be affected by changes in the configuration of the extracellular space and possible changes in endogenous protease activity (Kombian et al., 1997).

Release of the retrograde messengers OT and adenosine was shown previously to contribute to a positive feedback loop that results in a shift of activity of OT neurons from relative quiescence during pregnancy to synchronous bursting activity. Activity-induced somatodendritic release of $\mathrm{OT}$ and adenosine is important around parturition, as well as during lactation, when GABAergic inhibition of OT neurons is diminished by changes in $\mathrm{GABA}_{\mathrm{A}}$ receptor neurosteroid sensitivity we described previously (Brussaard et al., 1997). In this study, we show that release of OT and adenosine evoked by APs will further reduce GABAergic inhibition. The upregulation of the RRP and the increased calcium channel activity we describe in this report, together with the AP broadening, will increase the amount of OT and adenosine released per AP. This augments the reduction in GABAergic transmission during lactation. Increased extracellular OT may set in motion the positive feedback loop, described by Ludwig et al. (2002), and as a consequence, intracellular calcium stores will be activated via the OT receptor (Lambert et al., 1994) and stimulate additional release of OT and adenosine, which will affect neighboring neurons. The spread of disinhibition thus mediated is likely to result in synchronized activity of the OT neurons and eventually in milk letdown from the mammary glands.

In addition to the modulation of inhibitory synaptic input, there is also modulation of the glutamatergic synaptic inputs by oxytocin in the SON (Kombian et al., 1997; Hirasawa et al., 2001). It was shown that extracellular application of oxytocin or stimulation of the postsynaptic cells leads to a reduction in the amplitude of evoked but not miniature EPSCs. Modulation of both glutamatergic and GABAergic inputs seems counterintuitive but might serve to bring the SON neurons into the optimal voltage range of activity. The cells need to be disinhibited during the reproductive cycle, most likely via autoregulation of their GABA input (Brussaard et al., 1997; Brussaard and Herbison, 2000). However, without modulation of excitatory input, the positive autoregulation under such conditions may lead to overexcitation. We hypothesize that the plasticity of the autoregulatory mechanism and the subsequent physiological implications ensure the accurate timing of the various phases and the changing needs for OT release during the female reproductive cycle.

\section{References}

Armstrong WE, Stern JE, Teruyama R (2002) Plasticity in the electrophysiological properties of oxytocin neurons. Microsc Res Tech 56:73-80.

Bagdy E, Solyom S, Harsing Jr LG (1998) Feedback stimulation of somatodendritic serotonin release: a 5-HT3 receptor-mediated effect in the raphe nuclei of the rat. Brain Res Bull 45:203-208.

Brussaard AB, Herbison AE (2000) Long-term plasticity of postsynaptic $\mathrm{GABA}_{\mathrm{A}}$-receptor function in the adult brain: insights from the oxytocin neurone. Trends Neurosci 23:190-195.

Brussaard AB, Kits KS, de Vlieger TA (1996) Postsynaptic mechanism of depression of GABAergic synapses by oxytocin in the supraoptic nucleus of immature rat. J Physiol (Lond) 497:495-507.

Brussaard AB, Kits KS, Baker RE, Willems WP, Leyting-Vermeulen JW, Voorn P, Smit AB, Bicknell RJ, Herbison AE (1997) Plasticity in fast synaptic inhibition of adult oxytocin neurons caused by switch in $\mathrm{GABA}_{\mathrm{A}}$ receptor subunit expression. Neuron 19:1103-1114.

Brussaard AB, Devay P, Leyting-Vermeulen JL, Kits KS (1999) Changes in properties and neurosteroid regulation of GABAergic synapses in the supraoptic nucleus during the mammalian female reproductive cycle. J Physiol (Lond) 516:513-524.

Brussaard AB, Wossink J, Lodder JC, Kits KS (2000) Progesteronemetabolite prevents protein kinase C-dependent modulation of $\gamma$-aminobutyric acid type A receptors in oxytocin neurons. Proc Natl Acad Sci USA 97:3625-3630.

Bunin MA, Wightman RM (1998) Quantitative evaluation of 5-hydroxytryptamine (serotonin) neuronal release and uptake: an investigation of extrasynaptic transmission. J Neurosci 18:4854-4860.

Cheramy A, Leviel V, Glowinski J (1981) Dendritic release of dopamine in the substantia nigra. Nature 289:537-542.

Dudek FE, Kim YI, Bouskila Y (1993) Electrophysiology of the suprachiasmatic nucleus: synaptic transmission, membrane properties, and neuronal synchronization. J Biol Rhythms 8:S33-S37.

Falkenburger BH, Barstow KL, Mintz IM (2001) Dendrodendritic inhibition through reversal of dopamine transport. Science 293:2465-2470.

Hatton GI (1997) Function-related plasticity in hypothalamus. Annu Rev Neurosci 20:375-397.

Herrmann CS, Knight RT (2001) Mechanisms of human attention: eventrelated potentials and oscillations. Neurosci Biobehav Rev 25:465-476.

Hirasawa M, Kombian SB, Pittman QJ (2001) Oxytocin retrogradely inhibits evoked, but not miniature, EPSCs in the rat supraoptic nucleus: role of N- and P/Q-type calcium channels. J Physiol (Lond) 532:595-607.

Hou-Yu A, Lamme AT, Zimmerman EA, Silverman AJ (1986) Comparative distribution of vasopressin and oxytocin neurons in the rat brain using a double-label procedure. Neuroendocrinology 44:235-246.

Huang LY, Neher E (1996) $\mathrm{Ca}^{2+}$-dependent exocytosis in the somata of dorsal root ganglion neurons. Neuron 17:135-145.

Ivell R, Balvers M, Rust W, Bathgate R, Einspanier A (1997) Oxytocin and male reproductive function. Adv Exp Med Biol 424:253-264.

Jaffe EH, Marty A, Schulte A, Chow RH (1998) Extrasynaptic vesicular transmitter release from the somata of substantia nigra neurons in rat midbrain slices. J Neurosci 18:3548-3553.

Klyachko VA, Jackson MB (2002) Capacitance steps and fusion pores of small and large-dense-core vesicles in nerve terminals. Nature 418:89-92.

Kombian SB, Mouginot D, Pittman QJ (1997) Dendritically released peptides act as retrograde modulators of afferent excitation in the supraoptic nucleus in vitro. Neuron 19:903-912.

Lambert RC, Dayanithi G, Moos FC, Richard P (1994) A rise in the intracellular $\mathrm{Ca}^{2+}$ concentration of isolated rat supraoptic cells in response to oxytocin. J Physiol (Lond) 478:275-287.

Leng G, Brown CH, Russell JA (1999) Physiological pathways regulating the activity of magnocellular neurosecretory cells. Prog Neurobiol 57: $625-655$.

Lindau M, Neher E (1988) Patch-clamp techniques for time-resolved capacitance measurements in single cells. Pflügers Arch 411:137-146.

Link E, Edelmann L, Chou JH, Binz T, Yamasaki S, Eisel U, Baumert M, Sudhof TC, Niemann H, Jahn R (1992) Tetanus toxin action: inhibition of neurotransmitter release linked to synaptobrevin proteolysis. Biochem Biophys Res Commun 189:1017-1023.

Liu R, Jolas T, Aghajanian G (2000) Serotonin 5- $\mathrm{HT}_{2}$ receptors activate local GABA inhibitory inputs to serotonergic neurons of the dorsal raphe nucleus. Brain Res 873:34-45.

Ludwig M (1998) Dendritic release of vasopressin and oxytocin. J Neuroendocrinol 10:881-895.

Ludwig M, Sabatier N, Bull PM, Landgraf R, Dayanithi G, Leng G (2002) Intracellular calcium stores regulate activity-dependent neuropeptide release from dendrites. Nature 418:85-89.

Mansvelder HD, Kits KS (1998) The relation of exocytosis and rapid endocytosis to calcium entry evoked by short repetitive depolarizing pulses in rat melanotropic cells. J Neurosci 18:81-92.

Morin LP (1999) Serotonin and the regulation of mammalian circadian rhythmicity. Ann Med 31:12-33.

Neher E, Marty A (1982) Discrete changes of cell membrane capacitance observed under conditions of enhanced secretion in bovine adrenal chromaffin cells. Proc Natl Acad Sci USA 79:6712-6716.

Neumann I, Russell JA, Landgraf R (1993) Oxytocin and vasopressin release 
within the supraoptic and paraventricular nuclei of pregnant, parturient and lactating rats: a microdialysis study. Neuroscience 53:65-75.

Neumann I, Koehler E, Landgraf R, Summy-Long J (1994) An oxytocin receptor antagonist infused into the supraoptic nucleus attenuates intranuclear and peripheral release of oxytocin during suckling in conscious rats. Endocrinology 134:141-148.

Oliet SH, Poulain DA (1999) Adenosine-induced presynaptic inhibition of IPSCs and EPSCs in rat hypothalamic supraoptic nucleus neurones. J Physiol (Lond) 520:815-825.

Oliet SH, Piet R, Poulain DA (2001) Control of glutamate clearance and synaptic efficacy by glial coverage of neurons. Science 292:923-926.

Pow DV, Morris JF (1989) Dendrites of hypothalamic magnocellular neurons release neurohypophysial peptides by exocytosis. Neuroscience 32:435-439.

Radnikow G, Misgeld U (1998) Dopamine $\mathrm{D}_{1}$ receptors facilitate $\mathrm{GABA}_{\mathrm{A}}$ synaptic currents in the rat substantia nigra pars reticulata. J Neurosci 18:2009-2016.

Schiavo G, Benfenati F, Poulain B, Rossetto O, Polverino de Laureto P, DasGupta BR, Montecucco C (1992) Tetanus and botulinum-B neurotox- ins block neurotransmitter release by proteolytic cleavage of synaptobrevin. Nature 359:832-835.

Steriade M, Contreras D, Amzica F (1994) Synchronized sleep oscillations and their paroxysmal developments. Trends Neurosci 17:199-208.

Summerlee AJ (1981) Extracellular recordings from oxytocin neurones during the expulsive phase of birth in unanaesthetized rats. J Physiol (Lond) 321:1-9.

Theodosis DT (1985) Oxytocin-immunoreactive terminals synapse on oxytocin neurones in the supraoptic nucleus. Nature 313:682-684.

Theodosis DT, Poulain DA (1993) Activity-dependent neuronal-glial and synaptic plasticity in the adult mammalian hypothalamus. Neuroscience 57:501-535.

Theodosis DT, Chapman DB, Montagnese C, Poulain DA, Morris JF (1986) Structural plasticity in the hypothalamic supraoptic nucleus at lactation affects oxytocin-, but not vasopressin-secreting neurones. Neuroscience 17:661-678.

Theodosis DT, Bonfanti L, Olive S, Rougon G, Poulain DA (1994) Adhesion molecules and structural plasticity of the adult hypothalamo-neurohypophysial system. Psychoneuroendocrinology 19:455-462. 